\title{
Personalizing Cancer Therapy with FDG PET: From RECIST to PERCIST
}

W.A. Weber, guest editor

Reston, VA: Society of Nuclear Medicine, 2009, 150 pages, \$39
Monitoring therapeutic response is fundamental and crucial to clinical oncology because most chemotherapy drugs are effective in only subgroups of patients. Unfortunately, our current understanding of tumor biology does not allow us to predict accurately which patient may benefit from a specific therapeutic agent.

Various techniques have been developed for monitoring tumor response to cancer treatments, but measuring tumor shrinkage on CT remains the current standard method, using either a single linear summation (response evaluation criteria in solid tumors [RECIST]) or a bilinear product (World Health Organization criteria). However, the measurement based on anatomic imaging is being pressed to improve its methodologic robustness, particularly in light of more functional imaging such as ${ }^{18} \mathrm{~F}$-FDG PET that is sensitive to tissue molecular response.

The 12 articles in this supplement issue of The Journal of Nuclear Medicine are written by ${ }^{18}$ F-FDG PET experts throughout the world. The aim of the supplement, according to the guest editor, Dr. Weber, is to explore the clinical impact of PET on individualizing treatment regimens and also to describe the potential use of ${ }^{18} \mathrm{~F}-$ FDG PET in monitoring the response of various cancers to therapy. Each paper is intended to be a resource of currently available data on this topic. In addition, this supplement describes the clinical need to use ${ }^{18} \mathrm{~F}-\mathrm{FDG}$ PET for monitoring the response of specific conditions to therapy and provides guidance on practical issues such as the optimal timing of PET studies. It also describes the limitations of current studies of therapeutic monitoring with ${ }^{18}$ F-FDG PET.

After Dr. Weber's introductory article, Dr. Boellaard reviews ${ }^{18} \mathrm{~F}$-FDG PET protocols for data acquisition and analysis, as well as technical and biologic factors influencing standardized uptake value (SUV) measurements. In the following paper, Drs. Hutchings and Barrington discuss the current literature on ${ }^{18}$ F-FDG PET for monitoring the therapy of lymphoma. Many studies have indicated that ${ }^{18} \mathrm{~F}$-FDG PET is more accurate than CT for predicting the outcome of lymphoma patients after

COPYRIGHT (c) 2010 by the Society of Nuclear Medicine, Inc. DOI: 10.2967/jnumed.109.072934 chemotherapy. The next 6 papers summarize clinical experience with ${ }^{18} \mathrm{~F}-\mathrm{FDG}$ PET for monitoring treatments of common solid tumors (lung, colorectal, breast, cervical and ovarian, head and neck, and esophageal carcinomas). Their changes in glucose metabolic activities are generally smaller and also occur more slowly than in lymphomas because these solid tumors are more resistant to chemo- or radiotherapy. Therefore, even patients in whom ${ }^{18} \mathrm{~F}$-FDG PET shows a good response often demonstrate microscopic residual tumors after treatments, and thus the goal of ${ }^{18} \mathrm{~F}-\mathrm{FDG}$ PET is to guide decisions to intensify or change treatment in nonresponding patients.

New targeted cytostatic therapy may affect tumor glucose metabolism more directly and rapidly than cytotoxic treatment. Clinical experience on the use of ${ }^{18} \mathrm{~F}-\mathrm{FDG}$ PET for monitoring response to cytostatic treatment, reviewed by Drs. Contractor and Aboagye, appears limited except for treatment using imatinib. The papers in this supplement focus on ${ }^{18} \mathrm{~F}-\mathrm{FDG}$ because it represents the only PET agent clinically approved so far. Several other imaging probes targeting DNA synthesis, hypoxia, and amino acid metabolism, as well as estrogen and androgen receptors, are in the early stages of clinical development for monitoring tumor response to cancer therapy. These are discussed by Drs. Dunphy and Lewis. The supplement concludes with a proposal for PET response criteria in solid tumors (PERCIST) by Dr. Wahl and his colleagues. The premise of the PERCIST 1.0 criteria is a continuous and time-dependent variable, and the key elements of PERCIST include performance of PET scans in a method consistent with the National Cancer Institute recommendations. Response to therapy in PERCIST is expressed as percentage change in SUV corrected for lean body mass (SUL peak) or sum of SULs between the pre- and posttreatment scans. The SUL is determined for up to 5 tumors (up to 2 per organ) with the most intense ${ }^{18} \mathrm{~F}-\mathrm{FDG}$ uptake.

This supplement is an excellent review of updated information on monitoring therapeutic response with ${ }^{18} \mathrm{~F}-$ FDG PET and is useful for understanding current imaging techniques and their limitations. I highly recommend it to radiologists and oncologists, both in practice and in training, who need critical data and information on 
measuring tumor response to treatments. Nuclear physicians and scientists probably already received this supplement as members of the Society of Nuclear Medicine and should read the entire issue so that they can recommend the most appropriate technique for a given clinical situation and the optimal timing of follow-up ${ }^{18} \mathrm{~F}-\mathrm{FDG}$ PET studies. This supplement also provides insight on the need to continuously develop better methods and agents for the optimal prediction and monitoring of the response of cancer to therapy.

\section{E. Edmund Kim}

M.D. Anderson Cancer Center

1515 Holcombe Blvd., Unit 1264

Houston, TX 77030

E-mail:ekim@di.mdacc.tmc.edu 